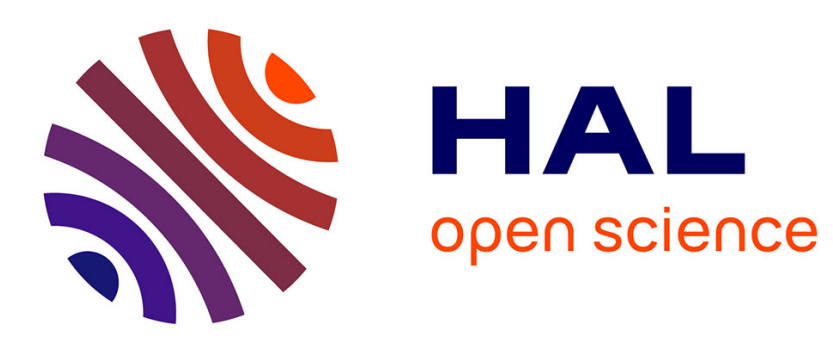

\title{
High gain observers with updated gain and homogeneous correction terms
}

Vincent Andrieu, Laurent Praly, Alessandro Astolfi

\section{To cite this version:}

Vincent Andrieu, Laurent Praly, Alessandro Astolfi. High gain observers with updated gain and homogeneous correction terms. Automatica, 2009, 45 (2), pp.422-428. 10.1016/j.automatica.2008.07.015 . hal-00362752

\section{HAL Id: hal-00362752 \\ https://hal.science/hal-00362752}

Submitted on 19 Feb 2009

HAL is a multi-disciplinary open access archive for the deposit and dissemination of scientific research documents, whether they are published or not. The documents may come from teaching and research institutions in France or abroad, or from public or private research centers.
L'archive ouverte pluridisciplinaire HAL, est destinée au dépôt et à la diffusion de documents scientifiques de niveau recherche, publiés ou non, émanant des établissements d'enseignement et de recherche français ou étrangers, des laboratoires publics ou privés. 


\title{
High gain observers with updated gain and homogeneous correction terms
}

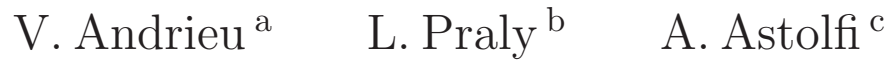 \\ ${ }^{\mathrm{a}}$ LAAS-CNRS, University of Toulouse, 31077 Toulouse, France \\ vincent.andrieu@gmail.com \\ ${ }^{\mathrm{b}} C A S$, École des Mines de Paris, Fontainebleau, France \\ praly@cas.ensmp.fr \\ ${ }^{\mathrm{c}}$ EEE Dept, Imperial College, London, UK and, \\ DISP, University of Rome Tor Vergata, Roma, Italy. a.astolfi@ic.ac.uk
}

\begin{abstract}
Exploiting dynamic scaling and homogeneity in the bi-limit, we developp a new class of high gain observers which incorporate a gain update law and nonlinear output error injection terms. A broader class of systems can be addressed and the observer gain is better fitted to the incremental rate of the nonlinearities. The expected improved performance is illustrated.
\end{abstract}

Key words: High-gain observers, Homogeneity in the bi-limit, Dynamic scaling.

\section{Introduction}

We extend the standard high-gain observer (see (Gauthier and Kupka, 2001) and references therein) in two directions : homogeneity and gain adaptation. Our motivation comes from considering the system :

$$
\dot{x}_{1}=x_{2}, \quad \dot{x}_{2}=f_{2}\left(x_{1}, x_{2}, u\right), \quad y=x_{1},
$$

with

$$
f_{2}\left(x_{1}, x_{2}, u\right)=g\left(x_{1}\right) x_{2}+x_{2}^{1+p}+u,
$$

where $p \geq 0$ is a real number, $g$ is a locally Lipschitz function and $u$ is a known input.

When $p=0$, we have :

$$
\left|f_{2}\left(x_{1}, x_{2}, u\right)-f_{2}\left(x_{1}, \hat{x}_{2}, u\right)\right| \leq\left|g\left(x_{1}\right)+1\right|\left|x_{2}-\hat{x}_{2}\right| \text {. }
$$

The term $\left|g\left(x_{1}\right)+1\right|$ is the output dependent incremental rate of the non-linearity. Systems with nonlinearities satisfying inequalities like (2) have already been studied in (Praly, 2003) (see also (Krishnamurthy et al., 2003)) and we know that a high gain observer can be used provided the gain is updated.

When $p$ is in the interval $(0,1)$, inequality $(2)$ becomes :

$$
\begin{array}{r}
\left|f_{2}\left(x_{1}, x_{2}, u\right)-f_{2}\left(x_{1}, \hat{x}_{2}, u\right)\right| \leq \\
\left(\left|g\left(x_{1}\right)\right|+(1+p)\left|\hat{x}_{2}\right|^{p}\right)\left|x_{2}-\hat{x}_{2}\right|+\left|x_{2}-\hat{x}_{2}\right|^{1+p} .
\end{array}
$$

The term, $\left|x_{2}-\hat{x}_{2}\right|^{1+p}$ is a rational power of the norm of the error $\left|x_{2}-\hat{x}_{2}\right|$. To deal with this term we use the homogeneous in the bi-limit observer introduced in (Andrieu et al., 2008-SICON).

In the following we address the problem of state observation for systems whose dynamics admit a global explicit observability canonical form (Gauthier and Kupka, 2001, Equation (20)) in which the nonlinearities have increments bounded as in (3). However, we restrict our attention to estimating the state only of those solutions which are bounded in positive time.

Our new observer uses a less conservative estimate of the nonlinearities increments. From this we expect the possibility of achieving better performance. This is confirmed via simulations of an academic model of a bioreactor.

\section{Main theoretical result}

We consider systems whose dynamics are :

$$
\left\{\begin{aligned}
\dot{x}_{1} & =f_{1}(u, y)+a_{1}(y) x_{2}+\delta_{1}(t), \\
& \vdots \\
\dot{x}_{i} & =f_{i}\left(u, y, x_{2}, \ldots, x_{i}\right)+a_{i}(y) x_{i+1}+\delta_{i}(t), \\
& \vdots \\
\dot{x}_{n} & =f_{n}\left(u, y, x_{2}, \ldots, x_{n}\right)+\delta_{n}(t), \\
y & =x_{1}+\delta_{y}(t),
\end{aligned}\right.
$$


where $y$ is the measured output in $\mathbb{R}$ and the functions $a_{i}$ and $f_{i}$ are locally Lipschitz. $u$ is a vector in $\mathbb{R}^{m}$ representing the known inputs and a finite number of their derivatives. The vector $\delta=\left(\delta_{1}, \ldots, \delta_{n}\right)$ represents the unknown inputs and $\delta_{y}$ is a measurement noise.

To simplify notations, let :

$$
w^{r}=\operatorname{sign}(w)|w|^{r} .
$$

so that, for instance, to recover the usual quadratic function we must write $\left|x^{2}\right|$ or $|x|^{2}$. We let also :

$$
\begin{aligned}
\mathcal{S} \cdot x & =\left(x_{2}, \ldots, x_{n}, 0\right)^{T}, \\
f(u, y, x) & =\left(f_{1}(u, y, x), \ldots, f_{n}(u, y, x)\right), \\
A(y) & =\operatorname{diag}\left(a_{1}(y), \ldots, a_{n}(y)\right),
\end{aligned}
$$

where $a_{n}$ is to be selected so that (5) below holds.

Theorem 1 Suppose there exists a continuous function $\mathfrak{a}$ satisfying, with $\rho, \underline{\mathfrak{A}}$ and $\overline{\mathfrak{A}}$ constant and for $j$ in $\{1, \ldots, n\}$,

$$
0<\rho \leq \mathfrak{a}(y), \quad 0<\underline{\mathfrak{A}} \leq \frac{a_{j}(y)}{\mathfrak{a}(y)} \leq \overline{\mathfrak{A}} \quad \forall y \in \mathbb{R},
$$

a real number $d_{\infty}$ in $\left[0, \frac{1}{n-1}\right)$, a positive real number $c_{\infty}$, a continuous function $\Gamma$ and real numbers $v_{j}$ in $\left[0, \frac{1}{j-1}\right)$, for $j=2, \ldots n$, such that, for all $i$ in $\{2, \ldots, n\}$ and all $(\hat{x}, x, y, u)$ in $\mathbb{R}^{n} \times \mathbb{R}^{n} \times \mathbb{R} \times \mathbb{R}^{m}$, we have :

$$
\begin{aligned}
& \left|f_{i}\left(u, y, \hat{x}_{2}, \ldots, \hat{x}_{i}\right)-f_{i}\left(u, y, x_{2}, \ldots, x_{i}\right)\right| \\
& \leq \Gamma(u, y)\left(1+\sum_{j=2}^{n}\left|\hat{x}_{j}\right|^{v_{j}}\right) \sum_{j=2}^{i}\left|\hat{x}_{j}-x_{j}\right| \\
& \quad+c_{\infty} \sum_{j=2}^{i}\left|\hat{x}_{j}-x_{j}\right|^{\frac{1-d_{\infty}(n-i-1)}{1-d_{\infty}(n-j)}} .
\end{aligned}
$$

Then, for all sufficiently small strictly positive real numbers $b$, there exists a function $K$ such that, for all sufficiently small strictly positive real number $\varphi_{1}$ and sufficiently large real numbers $\varphi_{2}$ and $\varphi_{3}$, we can find functions $\beta_{W}$ and $\beta_{L}$ of class $\mathcal{K} \mathcal{L}$ and functions $\gamma_{W}$ and $\gamma_{L}$ of class $\mathcal{K}$ such that the observer

$$
\begin{aligned}
& \dot{\hat{x}}=A(y) \mathcal{S} \hat{x}+f(u, y, \hat{x})+L \mathfrak{L} A(y) K\left(\frac{\hat{x}_{1}-y}{L^{b}}\right), \\
& \dot{L}=L\left[\varphi_{1}\left(\varphi_{2}-L\right)+\varphi_{3} \Omega(u, y, \hat{x})\right],
\end{aligned}
$$

with :

$$
\begin{gathered}
\Omega(u, y, \hat{x})=\Gamma(u, y)\left(1+\sum_{j=2}^{n}\left|\hat{x}_{j}\right|^{v_{j}}\right), \\
\mathfrak{L}=\operatorname{diag}\left(L^{b}, \ldots, L^{n+b-1}\right),
\end{gathered}
$$

initialized with $L(0) \geq \varphi_{2}$, has the following property: For each solution $t \mapsto x(t)$ of (4) right maximally defined on $[0, \mathcal{T})$, the observer solution is defined on the same interval and the error estimate $\mathfrak{e}=\hat{x}-x$ satisfies :

$\left|\mathfrak{L}(t)^{-1} \mathfrak{e}(t)\right| \leq \beta_{W}\left(\mathfrak{L}(0)^{-1} \mathfrak{e}(0), t\right)$

$$
+\sup _{s \in[0, t]} \gamma_{W}\left(\left|\left(\begin{array}{c}
\frac{\delta(s)}{\varphi_{2}} \\
\mathfrak{a}(y(s)) \delta_{y}(s)
\end{array}\right)\right|\right) \quad \forall t \in[0, \mathcal{T})
$$

where L satisfies :

$$
\begin{aligned}
& L(t) \leq 4 \varphi_{2}+\beta_{L}\left(\left(\begin{array}{c}
\mathfrak{e}(0) \\
L(0)
\end{array}\right), t\right) \\
& +\sup _{s \in[0, t]} \gamma_{L}\left(\mid\left(\begin{array}{c}
\frac{\delta(s)}{\varphi_{2}} \\
\mathfrak{a}(y(s)) \delta_{y}(s) \\
\Gamma(u(s), y(s)) \\
x(s)
\end{array}\right)\right) \text {. }
\end{aligned}
$$

\subsection{Discussion on the assumptions}

The form (4) is a particular case of the implicit form obtained in (Gauthier and Kupka, 2001, Equation (20)). The functions $a_{i}$ and $f_{i}$ in (4), are not uniquely defined. We can get other functions by changing coordinates and, in this way, possibly satisfy conditions (6).

To understand the meaning of (6), we observe that, for any $C^{1}$ function $f$, there exist always two functions $\mho$ and $\Delta$ such that we have :

$$
|f(a, b+c)-f(a, b)| \leq \mho(a, b)|c|+\Delta(c) .
$$

Hence, in essence, (6) imposes two restrictions :

- the function $\Omega$, defined in (9), is a bound on the local incremental rate $\mho \delta$.

- a fractional power limitation, $\frac{1-d_{\infty}(n-i-1)}{1-d_{\infty}(n-j)}$ with $d_{\infty}$ in $\left[0, \frac{1}{n-1}\right)$, on the growth of $\Delta$ which bounds function increments for large argument increments.

For system (1), inequality (3) is in the form (6) with $d_{\infty}=p, \Gamma(u, y)=(|g(y)|+1+p)$ and $v_{2}=p$. Hence, Theorem 1 applies when $p$ is in the interval $[0,1)$. Actually, when $p>1$ and $u=0$, there does not exist any observer guaranteeing convergence of the estimation error within the domain of existence of the solutions (see (Astolfi and Praly, 2006, Proposition 1)).

\subsection{Discussion on the result}

With (11) and (12) but with the presence of $\sup _{s}|x(s)|$, Theorem 1 says that the observer $(7),(8)$ gives, at least for bounded solutions, an estimation error converging to a ball centered at the origin and with radius depending on the asymptotic $L^{\infty}$-norm of the disturbances $\delta$ and $\delta_{y}$ and therefore converging to the origin if these disturbances are vanishing.

Although we restrict our attention to bounded solutions, we are not back to the global Lipschitz case since the "Lipschitz constant" is solution dependent and therefore unavailable for observer design. It has to be learned on line and this is what $L$ is doing in (8). The update law for $L$ is very similar to the one introduced in (Praly, 2003) (see also (Krishnamurthy et al., 2003)). The difference is in the fact that (8) depends also on $\hat{x}$ and $u$ and not 
only on $y$ and we need the restrictions on $v_{j}$ to deal with this dependence on $\hat{x}$.

If $\Omega$ were differentiable along the solutions, the update law (8) would give :

$$
\overbrace{L-\left(\varphi_{2}+\frac{\varphi_{3}}{\varphi_{1}} \Omega\right)}=\frac{\varphi_{3}}{\varphi_{1}} \dot{\Omega}-\varphi_{1} L\left[L-\left(\varphi_{2}+\frac{\varphi_{3}}{\varphi_{1}} \Omega\right)\right]
$$

This says that $L$ would track $\varphi_{2}+\frac{\varphi_{3}}{\varphi_{1}} \Omega$ up to an error proportional to the magnitude of $\dot{\Omega}$. We expect improved performance from this tracking property (see Section 3 ).

\subsection{Comparison with published results}

High gain observers have a long history. The prototype result is (Gauthier and Kupka, 2001, Theorem 6.2.2). It deals with systems admitting an observability canonical representation more general than (4) by being implicit in $x_{i+1}$. But there the right hand side of inequality (6) is supposed to be $\Gamma \sum_{j=2}^{i}\left|\hat{x}_{j}-x_{j}\right|$ with $\Gamma$ constant.

The case where $\Gamma$ may depend on $u$ and $y$ can be handled with updating the gain as in (8). This extends what can be found in (Praly, 2003) when the $a_{i}$ are constant and in (Krishnamurthy et al., 2003) when the $a_{i}$ are $y$ dependent.

The idea of having homogeneous (in the classical weighted sense) correction terms has been introduced in (Qian, 2005) for a pure chain of integrator, i.e. when the $a_{i}$ 's are constant and the $f_{i}$ are zero.

Another observer is proposed in (Lei et al., 2005), for systems with bounded solutions and admitting the same form (4) with the $a_{i}$ 's constant and $f_{1}=\ldots=f_{n-1}=0$ but with no restriction on $f_{n}$. However this is obtained by having a gain which grows monotonically with time along the solutions.

\section{Discussion and illustration}

To illustrate the interest for applications of our observer and the tracking property noticed in (13), we consider the same "academic" bioreactor as the one studied in (Gauthier et al., 1992). Its dynamics are described, in normalized variables and time, by the Contois model :

$\dot{\eta}_{1}=\frac{\eta_{1} \eta_{2}}{\hbar \eta_{1}+\eta_{2}}-u \eta_{1}, \quad \dot{\eta}_{2}=-\frac{\eta_{1} \eta_{2}}{\hbar \eta_{1}+\eta_{2}}+u\left(1-\eta_{2}\right)$

where $y=\eta_{1}$ is measured. The parameter $\hbar$ is a positive real number and the control input $u$ is in the interval $\mathcal{M}_{u}=\left[u_{\min }, u_{\max }\right] \subset(0,1)$. In (Gauthier et al., 1992), it is observed that the following set is forward invariant: $\mathcal{M}_{\eta}=\left\{\left(\eta_{1}, \eta_{2}\right) \in \mathbb{R}^{2}: \eta_{1} \geq \epsilon_{1}, \eta_{2} \geq \epsilon_{2}, \eta_{1}+\eta_{2} \leq 1\right\}$, where, $\epsilon_{1}=\frac{\left(1-u_{\max }\right) \epsilon_{2}}{\hbar u_{\max }}$, and $u_{\min } \geq \frac{\epsilon_{2}}{\hbar\left(1-\epsilon_{2}\right)+\epsilon_{2}}$. This guarantees that the bioreactor state remains in a known compact set.
Following (Gauthier et al., 1992), we change the coordinates as :

$$
\left(\eta_{1}, \eta_{2}\right) \mapsto\left(x_{1}, x_{2}\right)=F\left(\eta_{1}, \eta_{2}\right)=\left(\eta_{1}, \frac{\eta_{1} \eta_{2}}{\hbar \eta_{1}+\eta_{2}}\right) .
$$

with $x$ evolving in $\mathcal{M}_{x}=F\left(\mathcal{M}_{\eta}\right)$. In these new coordinates the system is in the explicit observability canonical form :

$$
\dot{x}_{1}=x_{2}-u x_{1}, \quad \dot{x}_{2}=f_{2}\left(x_{1}, x_{2}, u\right), \quad y=\eta_{1},
$$
with,

$$
f_{2}\left(x_{1}, x_{2}, u\right)=m_{0}+m_{1} x_{2}+m_{2} x_{2}^{2}+m_{3} x_{2}^{3}
$$
where :

$$
\begin{aligned}
& m_{0}=\frac{u}{\hbar} \quad, \quad m_{1}=-u-\frac{1}{\hbar}-\frac{2 u}{\hbar x_{1}}, \\
& m_{2}=\frac{2}{\hbar x_{1}}+\frac{u}{\hbar x_{1}^{2}} \quad, \quad m_{3}=\frac{\hbar-1}{\hbar x_{1}^{2}} .
\end{aligned}
$$

Note that, for all $\left(x_{1}, x_{2}, u\right)$ in $\mathcal{M}_{x} \times \mathcal{M}_{u}$, we have :

$$
\underline{x}_{2}\left(x_{1}\right)=x_{1} \frac{\epsilon_{2}}{\hbar x_{1}+\epsilon_{2}} \leq x_{2} \leq x_{1} \frac{1-x_{1}}{1-x_{1}+\hbar x_{1}}=\bar{x}_{2}\left(x_{1}\right) .
$$

Hence, without loss of generality, to evaluate $f_{2}$ in (15), we can replace $\left(x_{1}, x_{2}\right)$ by $\left(x_{1 s}, x_{2 s}\right)$ defined as

$$
\begin{aligned}
& x_{1 s}=\max \left\{\epsilon_{1}, \min \left\{1-\epsilon_{2}, x_{1}\right\}\right\}, \\
& x_{2 s}=\max \left\{\underline{x}_{2}\left(x_{1 s}\right), \min \left\{\bar{x}_{2}\left(x_{1 s}\right), x_{2}\right\}\right\}
\end{aligned}
$$

and therefore assume that $f_{2}$ is globally Lipschitz.

For a nominal high gain observer, as in (Gauthier et al., 1992), the nonlinearity increment is bounded as :

$$
\left|f_{2}\left(x_{1}, x_{2}, u\right)-f_{2}\left(x_{1}, \hat{x}_{2}, u\right)\right| \leq d f_{2 \max }\left|x_{2}-\hat{x}_{2}\right| .
$$

where, from the Mean Value Theorem,

$$
d f_{2 \max }=\max _{\left(u, x_{1}, x_{2}\right) \in \mathcal{M}_{u} \times \mathcal{M}_{x}}\left|m_{1}+2 m_{2} x_{2}+3 m_{3} x_{2}^{2}\right| .
$$

For an updated high gain observer, the bound is :

$\left|f_{2}\left(x_{1}, x_{2}, u\right)-f_{2}\left(x_{1}, \hat{x}_{2}, u\right)\right| \leq \Omega_{1}\left(u, x_{1}, \hat{x}_{2}\right)\left|x_{2}-\hat{x}_{2}\right|$, with

$$
\begin{aligned}
& \Omega_{1}\left(u, x_{1}, \hat{x}_{2}\right)= \\
& \max _{x_{2} \in\left[\underline{x}_{2}\left(x_{1 s}\right), \bar{x}_{2}\left(x_{1 s}\right)\right]}\left|m_{1}+\left[m_{2}+m_{3} \hat{x}_{2}\right]\left(\hat{x}_{2}+x_{2}\right)+m_{3} x_{2}^{2}\right| . \\
& \hat{x}_{2 s}=\max \left\{\underline{x}_{2}\left(x_{1 s}\right), \min \left\{\bar{x}_{2}\left(x_{1 s}\right), \hat{x}_{2}\right\}\right\} .
\end{aligned}
$$

It follows that Theorem 1 applies with $d_{\infty}=c_{\infty}=0$.

Finally for our observer with both updated gain and rational power error term, the bound is :

$$
\begin{aligned}
& \left|f_{2}\left(x_{1}, x_{2}, u\right)-f_{2}\left(x_{1}, \hat{x}_{2}, u\right)\right| \\
& \leq \Omega_{2}\left(u, x_{1}, \hat{x}_{2}\right)\left|x_{2}-\hat{x}_{2}\right|+c_{\infty}\left|x_{2}-\hat{x}_{2}\right|^{1+p},
\end{aligned}
$$

with $p$ in $(0,1)$ and where

$$
\begin{aligned}
& \Omega_{2}\left(u, x_{1}, \hat{x}_{2}\right)=\max _{x_{2} \in\left[\underline{x}_{2}\left(x_{1 s}\right), \bar{x}_{2}\left(x_{1 s}\right)\right]} \\
& \quad \begin{array}{l}
\left.\left.\mid m_{1}+\hat{x}_{2}^{p}\left(\left[m_{2}+m_{3} \hat{x}_{2}\right]\left[\hat{x}_{2}^{1-p}+x_{2}^{1-p}\right]+m_{3} x_{2}^{2-p}\right)\right]\right) \mid \\
c_{\infty}=\max _{\left(u, x_{1}, x_{2}, \hat{x}_{2}\right) \in \mathcal{M}_{u} \times \mathcal{M}_{x} \times\left[\underline{x}_{2}\left(\epsilon_{1}\right), \bar{x}_{2}\left(1-\epsilon_{2}\right)\right]} \\
\left|\left(m_{2}+m_{3} \hat{x}_{2}\right) x_{2}^{1-p}+m_{3} x_{2}^{2-p}\right|
\end{array}
\end{aligned}
$$




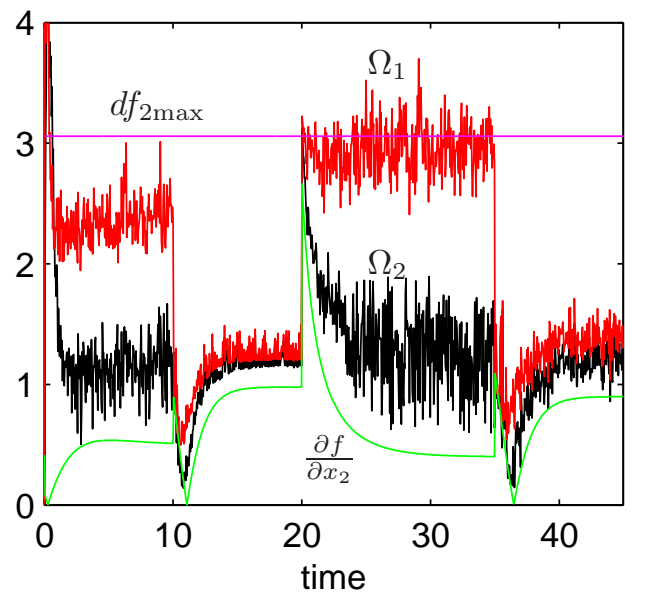

Fig. 1. Approximations of the local incremental rates

In this case, Theorem 1 gives the following observer :

$$
\left\{\begin{array}{c}
\dot{\hat{x}}_{1}=\hat{x}_{2}-u y-L^{1+b} q_{1}\left(\ell_{1} \frac{\left[\hat{x}_{1}-y\right]}{L^{b}}\right), \\
\dot{\hat{x}}_{2}=f_{2}\left(y, \hat{x}_{2 s}, u\right) \\
\quad-L^{2+b} q_{2}\left(\ell_{2} q_{1}\left(\ell_{1} \frac{\left[\hat{x}_{1}-y\right]}{L^{b}}\right)\right), \\
\dot{L}=L\left[\varphi_{1}\left(\varphi_{2}-L\right)+\varphi_{3} \Omega_{2}\left(u, y, \hat{x}_{2 s}\right)\right],
\end{array}\right.
$$

where $q_{1}(s)=s+s^{\frac{1}{1-p}}, q_{2}(s)=s+s^{1+p}$ and $b, \varphi_{i}$ and $\ell_{i}$ are parameters to be chosen.

Since we have, for all $\left(x_{1}, x_{2}, u\right)$ in $\mathcal{M}_{x} \times \mathcal{M}_{u}$,

$\frac{\partial f_{2}}{\partial x_{2}}\left(x_{1}, x_{2}, u\right) \leq \Omega_{2}\left(u, x_{1}, x_{2}\right) \leq \Omega_{1}\left(u, x_{1}, x_{2}\right) \leq d f_{2 \max }$

we expect the updated high gain observer to give better performance than the one without adaptation, and the new one proposed in this paper to give even better behavior in particular in presence of measurement noise.

\subsection{Simulations}

We illustrate the behavior of the observers with simulations. But this is no more than an illustration and we do not claim that our observer is the best one for this particular application ${ }^{1}$.

The control input is selected as :

$$
\begin{aligned}
& u(t)=0.410 \text { if } t<10, \quad=0.02 \text { if } 10 \leq t<20, \\
& =0.6 \quad \text { if } 20 \leq t<35,=0.1 \text { if } 35 \leq t .
\end{aligned}
$$

From this we have chosen $u_{\min }=0.01$ and $u_{\max }=0.7$ and $\epsilon_{1}$ and $\epsilon_{2}$ accordingly. Also, we have introduced two disturbances :

- the measurement disturbance is a Gaussian white

1 A simple copy (without correction term) gives an observer which is not sensitive to measurement noise but on the other hand we cannot assign its speed of convergence.
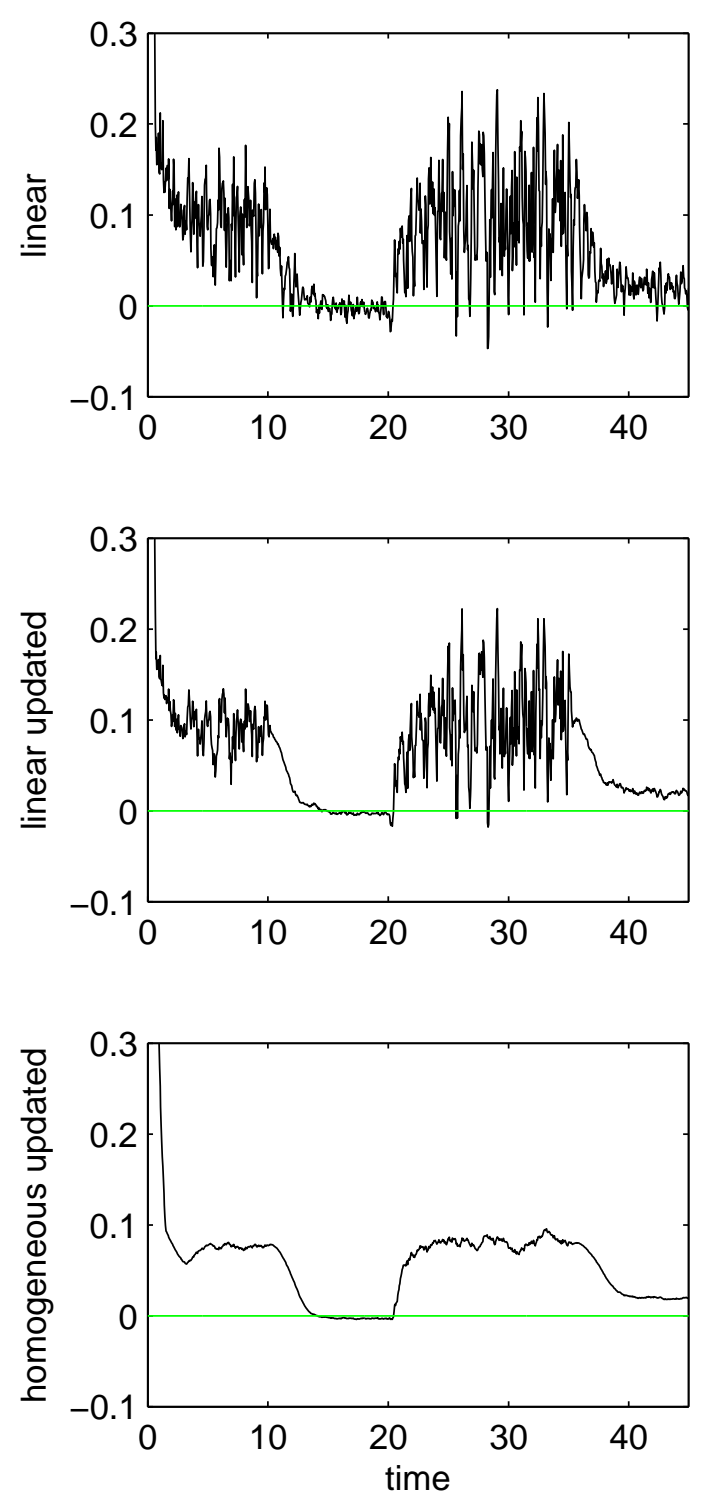

Fig. 2. Estimation error $\eta_{2}-\hat{\eta}_{2}$ given by each observer.

noise with standard deviation equal to $10 \%$ of the $\eta_{1}$ domain $\left[\epsilon_{1}, 1-\epsilon_{2}\right]$, i.e. $=0.05$.

- a $20 \%$ error in $\hbar$. The value used for the system (14)

is 1 , whereas the one in the observers is 0.8

For the observers we have used the following values :

$$
p=0.9, b=0.410
$$

$$
\varphi_{1}=0.03, \varphi_{2}=1, \varphi_{3}=3, \ell_{1}=0.01, \ell_{2}=0.01 \text {. }
$$

Figure 1 shows the values of the estimates of the local incremental rate of $f_{2}$ (i.e. $\left.\frac{\partial f_{2}}{\partial x_{2}}\right), d f_{2 \max }$ for the high-gain observer, $\Omega_{1}$ for an updated high-gain observer, and $\Omega_{2}$ for a homogeneous updated high-gain observer. In spite of the measurement noise, the predicted order $d f_{2 \max } \geq$ $\Omega_{1} \geq \Omega_{2} \geq \frac{\partial f_{2}}{\partial x_{2}}$ is observed in the mean. 
Figure 2 displays the plot of the estimation error $\eta_{2}-\hat{\eta}_{2}$, given by the observers with constant gain deduced from $d f_{2 \text { max }}$ (top), with adapted gain deduced from $\Omega_{1}$ (middle), and with adapted gain deduced from $\Omega_{2}$ and homogeneity (bottom). In the three cases there is a bias, due to the error in $\hbar$, which increases with the estimates of the local incremental rate. We see also a strong correlation between the standard deviation of the error $\hat{\eta}_{2}-\eta_{2}$ and the magnitude of these estimates respectively used, i.e. $d f_{2 \max }, \Omega_{1}$ and $\Omega_{2}$. As expected the best result is given by the new observer based on $\Omega_{2}$.

\section{Proof of Theorem 1}

Theorem 1 is proved in Subsection 4.3. It needs some prerequisite summarized now and which can be found in (Andrieu et al., 2008-SICON).

\subsection{Homogeneous approximation}

Given a vector $r=\left(r_{1}, \ldots, r_{n}\right)$ in $\left(\mathbb{R}_{+} /\{0\}\right)^{n}$, we define the dilation of a vector $x$ in $\mathbb{R}^{n}$ as

$$
\lambda^{r} \diamond x=\left(\lambda^{r_{1}} x_{1}, \ldots, \lambda^{r_{n}} x_{n}\right)^{T} .
$$

\section{Definition 1}

- A continuous function $\phi: \mathbb{R}^{n} \rightarrow \mathbb{R}$ is said homogeneous in the 0-limit (respectively $\infty$-limit) with associated triple $\left(r_{0}, d_{0}, \phi_{0}\right)$ (resp. $\left.\left(r_{\infty}, d_{\infty}, \phi_{\infty}\right)\right)$, where $r_{0}$ (resp. $\left.r_{\infty}\right)$ in $\left(\mathbb{R}_{+} /\{0\}\right)^{n}$ is the weight, $d_{0}$ $\left(\right.$ resp. $\left.d_{\infty}\right)$ in $\mathbb{R}_{+}$the degree and $\phi_{0}: \mathbb{R}^{n} \rightarrow \mathbb{R}$ (resp. $\phi_{\infty}: \mathbb{R}^{n} \rightarrow \mathbb{R}$ ) the approximating function, if $\phi_{0}$ (resp. $\left.\phi_{\infty}\right)$ is continuous and not identically zero and, for each compact set $C$ in $\mathbb{R}^{n}$ and each $\varepsilon>0$, there exists $\lambda^{*}$ such that we have :

$$
\begin{aligned}
& \max _{x \in C}\left|\frac{\phi\left(\lambda^{r_{0}} \diamond x\right)}{\lambda^{d_{0}}}-\phi_{0}(x)\right| \leq \varepsilon \quad \forall \lambda \in\left(0, \lambda^{*}\right] . \\
& \text { (respectively } \\
& \left.\max _{x \in C}\left|\frac{\phi\left(\lambda^{r_{\infty}} \diamond x\right)}{\lambda^{d_{\infty}}}-\phi_{\infty}(x)\right| \leq \varepsilon \quad \forall \lambda \in\left[\lambda^{*},+\infty\right) .\right)
\end{aligned}
$$

- $A$ vector field $f=\sum_{i=1}^{n} f_{i} \frac{\partial}{\partial x_{i}}$ is said homogeneous in the 0-limit (resp. $\infty$-limit) with associated triple $\left(r_{0}, d_{0}, f_{0}\right)$ (resp. $\left.\left(r_{\infty}, d_{\infty}, f_{\infty}\right)\right)$, where $f_{0}=\sum_{i=1}^{n} f_{0, i} \frac{\partial}{\partial x_{i}}$ (resp. $\left.f_{\infty}=\sum_{i=1}^{n} f_{\infty, i} \frac{\partial}{\partial x_{i}}\right)$, if, for each $i$ in $\{1, \ldots, n\}$, the function $f_{i}$ is homogeneous in the 0-limit (resp. $\infty$-limit) with associated triple $\left(r_{0}, d_{0}+r_{0, i}, f_{0, i}\right)^{2}$.

Definition 2 A continuous function $\phi: \mathbb{R}^{n} \rightarrow \mathbb{R}$ (or a vector field $f$ ) is said homogeneous in the bi-limit if it is homogeneous in the 0 -limit and in the $\infty$-limit.

\footnotetext{
${ }^{2}$ In the case of a vector field the degree $d_{0}$ can be negative as long as $d_{0}+r_{0, i} \geq 0$ (resp. $\left.\left(r_{\infty}, d_{\infty}+r_{\infty, i}, f_{\infty, i}\right)\right)$, for all $1 \leq i \leq n$.
}

\subsection{Homogeneous in the bi-limit observer}

Consider the following chain of integrators on $\mathbb{R}^{n}$ :

$$
\dot{\mathfrak{X}}=\mathfrak{A}(t) \mathcal{S} \mathfrak{X},
$$

where $\mathfrak{A}(t)=\operatorname{diag}\left(\mathfrak{A}_{1}(t), \ldots, \mathfrak{A}_{n}(t)\right)$, is a known time varying matrix with the $\mathfrak{A}_{i}$ satisfying, with $\underline{\mathfrak{A}}$ and $\overline{\mathfrak{A}}$ constant,

$$
0<\underline{\mathfrak{A}} \leq \mathfrak{A}_{i}(t) \leq \overline{\mathfrak{A}} \quad \forall t .
$$

With $d_{0}=0$ and $d_{\infty}$ arbitrary in $\left[0, \frac{1}{n-1}\right)$, the system (16) is homogeneous in the bi-limit with the weights $r_{0}=\left(r_{0,1}, \ldots, r_{0, n}\right)$ and $r_{\infty}=\left(r_{\infty, 1}, \ldots, r_{\infty, n}\right)$ as :

$$
r_{0, i}=1, r_{\infty, i}=1-d_{\infty}(n-i) .
$$

In (Andrieu et al., 2008-SICON), a new observer was proposed for system (16) for the particular case where $\mathfrak{A}_{i}(t)=1$. Its design is done recursively together with the one of an appropriate error Lyapunov function $W$ which is homogeneous in the bi-limit.

To combine this tool with gain updating we need an extra property on $W$ which is a counterpart of (Praly, 2003, equation (16)) or (Krishnamurthy et al., 2003, Lemma A1). We have :

Theorem 2 Let $d_{\infty}$ be in $\left[0, \frac{1}{n-1}\right), d_{W}$ in $\left[2+d_{\infty}, \infty\right)$ and $\mathfrak{B}=\operatorname{diag}\left(\mathfrak{b}_{1}, \ldots, \mathfrak{b}_{n}\right)$ with $\mathfrak{b}_{j}>0$. If (17) holds, there exist a vector field $K: \mathbb{R} \rightarrow \mathbb{R}^{n}$ which is homogeneous in the bi-limit with associated weights $r_{0}$ and $r_{\infty}$, and a positive definite, proper and $C^{1}$ function $W: \mathbb{R}^{n} \rightarrow$ $\mathbb{R}_{+}$, homogeneous in the bi-limit with associated triples $\left(r_{0}, d_{W}, W_{0}\right)$ and $\left(r_{\infty}, d_{W}, W_{\infty}\right)$, such that

(1) The functions $W_{0}$ and $W_{\infty}$ are positive definite and proper and, for each $j$ in $\{1, \ldots, n\}$, the function $\frac{\partial W}{\partial e_{j}}$ is homogeneous in the bi-limit with approximating functions $\frac{\partial W_{0}}{\partial e_{j}}$ and $\frac{\partial W_{\infty}}{\partial e_{j}}$.

(2) There exist two positive real numbers $c_{1}$ and $c_{2}$ such that we have, for all $(t, E)$ in $\mathbb{R} \times \mathbb{R}^{n}$,

$$
\begin{aligned}
\frac{\partial W}{\partial E}(E) \mathfrak{A}( & t)\left(\mathcal{S} E+K\left(e_{1}\right)\right) \\
\leq & -c_{1}\left(W(E)+W(E)^{\frac{d_{W}+d_{\infty}}{d_{W}}}\right) \\
\frac{\partial W}{\partial E}(E) \mathfrak{B} E & \geq c_{2} W(E) .
\end{aligned}
$$

For proving this result, the only difference compared with what is done in (Andrieu et al., 2008-SICON) is to multiply $W_{i}$ by a sufficiently small positive real number $\sigma_{i}$ before using it in the definition of $W_{i-1}$. The proof is omitted due to space limitation. It can be found in (Andrieu et al., 2008-HAL).

\subsection{Proof of Theorem 1}

Let $\mathfrak{A}$ and $\mathfrak{B}$ in Theorem 2 be (see (Praly, 2003)) : 
$\mathfrak{A}(t)=\frac{A(y(t))}{\mathfrak{a}(y(t))}, \quad \mathfrak{B}=\operatorname{diag}(b, 1+b, \ldots, n-1+b)^{T}$,

where $y(t)$ is the evaluation of $y$ along a solution and $b$ is a positive real number satisfying, for all $1 \leq j \leq i \leq n$,

$$
\begin{gathered}
\frac{1-d_{\infty}(n-i-1)}{1-d_{\infty}(n-j)}<\frac{i+b}{j-1+b}<\frac{i}{j-1}, \\
\text { and } \quad 0<b<\frac{1-v_{j}(j-1)}{v_{j}},
\end{gathered}
$$

with $d_{\infty}$ and $v_{j}$ as given in the statement of Theorem 1.

From Theorem 2, we obtain a homogeneous in the bilimit vector field $K$ and a homogeneous in the bi-limit Lyapunov function $W$ satisfying (19) and (20). This allows us to write the observer as in (7)-(8). Note that if $L(0) \geq \varphi_{2}$ then $L(t) \geq \varphi_{2}$ for all $t \geq 0$ in the domain of definition.

Properties of the estimation error. With $\mathfrak{L}$ given in (10), let $E=\left(e_{1}, \ldots, e_{n}\right)$ and $\tau$ be defined as :

$$
E=\mathfrak{L}^{-1}(\hat{x}-x)=\mathfrak{L}^{-1} \mathfrak{e} \quad, \quad d \tau=L d t .
$$

Since we have :

we get :

$$
\overbrace{\mathfrak{L}^{-1}}^{\cdot}=-L^{-1} \dot{L} \mathfrak{B} \mathfrak{L}^{-1}
$$

$$
\begin{array}{r}
\frac{d E}{d \tau}=A(y)\left[\mathcal{S} E+K\left(e_{1}-\frac{\delta_{y}}{L^{b}}\right)\right]-L^{-1} \mathfrak{L}^{-1} \delta \\
+\mathfrak{D}(L)-L^{-1} \frac{d L}{d \tau} \mathfrak{B} E
\end{array}
$$

with $\mathfrak{D}(L)$ defined as

$$
\mathfrak{D}(L)=\left(\ldots, \frac{f_{i}(u, y, \hat{x})-f_{i}(u, y, x)}{L^{i+b}}, \ldots\right) .
$$

Along the solutions of the system (24) we have :

$$
\begin{aligned}
\frac{d W(E)}{d \tau}=\frac{\partial W}{\partial E}(E) \mathfrak{a}(y) & \mathfrak{A}(t)\left[\mathcal{S} E+K\left(e_{1}\right)\right] \\
+ & T_{\delta}+T_{y}+T_{G U}+T_{N L},
\end{aligned}
$$

with the notations

$$
\begin{aligned}
T_{\delta} & =-\frac{\partial W}{\partial E}(E) L^{-1} \mathfrak{L}^{-1} \delta \\
T_{y} & =\frac{\partial W}{\partial E}(E) \mathfrak{a}(y) \mathfrak{A}(t)\left[K\left(e_{1}-\frac{\delta_{y}}{L^{b}}\right)-K\left(e_{1}\right)\right], \\
T_{G U} & =-L^{-2} \dot{L} \frac{\partial W}{\partial E}(E) \mathfrak{B} E \\
T_{N L} & =\frac{\partial W}{\partial E}(E) \mathfrak{D}(L),
\end{aligned}
$$

and, with (19), we have :

$$
\begin{aligned}
& \frac{\partial W}{\partial E}(E) \mathfrak{a}(y) \mathfrak{A}(t)\left(\mathcal{S} E+K\left(e_{1}\right)\right) \\
& \leq-c_{1} \mathfrak{a}(y)\left(W(E)+W(E)^{\frac{d_{W}+d_{\infty}}{d_{W}}}\right)
\end{aligned}
$$

Bounding $T_{\delta}$. With weights 1 and $r_{\infty, i}+d_{\infty}$ for $\frac{\delta_{i}}{L^{i}}$, the function $\left(E, \frac{\delta_{i}}{L^{i}}\right) \mapsto \frac{\partial W}{\partial e_{i}}(E) \frac{\delta_{i}}{L^{i}}$ is homogeneous in the bi-limit with degrees $d_{W}$ and $d_{W}+d_{\infty}$. Also $W, W_{0}$ and $W_{\infty}$ are positive definite. Hence (see (Andrieu et al., 2008-SICON, Corollary 2.15)) there exists a positive real number $c_{3}$ satisfying, for any $E, i$ and $L \geq \varphi_{2}$,

$$
\begin{aligned}
\frac{\partial W}{\partial e_{i}}(E) \frac{\delta_{i}}{L^{i+b}} \leq \frac{c_{3}}{n \varphi_{2}^{b}}\left[W(E)+W(E)^{\frac{d_{W}+d_{\infty}}{d_{W}}}\right] & \\
& +\frac{c_{3}}{n L^{b}} \mathfrak{H}_{i}\left(\frac{\delta_{i}}{L^{i}}\right) .
\end{aligned}
$$

where $\mathfrak{H}_{i}: \mathbb{R}_{+} \rightarrow \mathbb{R}_{+}$is the strictly increasing, homogeneous in bi-limit function defined as

$$
\mathfrak{H}_{i}(s)=\left(1+|s|^{\frac{d_{W}+d_{\infty}}{r_{\infty}+d_{\infty}}}\right) \frac{|s|^{d_{W}}}{1+|s|^{d_{W}}}
$$

This gives :

$T_{\delta} \leq \frac{c_{3}}{\varphi_{2}^{b}}\left[W(E)+W(E)^{\frac{d_{W}+d_{\infty}}{d_{W}}}\right]+\frac{c_{3}}{n L^{b}} \sum_{i=1}^{n} \mathfrak{H}_{i}\left(\frac{\delta_{i}}{L^{i}}\right)$.

Bounding $T_{y}$. Let $k_{i}$ be the $i$ th component of $K$ and $\mu$ and $\eta$ be the functions :

$$
\begin{gathered}
\mu(\bar{\delta})=|\bar{\delta}|^{d_{W}}+|\bar{\delta}|^{\frac{d_{W}+d_{\infty}}{r_{\infty}, 1}} \\
\eta(E, \bar{\delta})=\frac{c_{1}}{4 n \overline{\mathfrak{A}}}\left[W(E)+W(E)^{\frac{d_{W}+d_{\infty}}{d_{W}}}\right] \\
-\frac{\partial W}{\partial e_{i}}(E)\left[k_{i}\left(e_{1}+\bar{\delta}\right)-k_{i}\left(e_{1}\right)\right]
\end{gathered}
$$

From the properties of $K$, with the weights 1 and $r_{\infty, 1}$ for $\bar{\delta}$, the functions $\bar{\delta} \mapsto \mu(\bar{\delta})$ and $(E, \bar{\delta}) \mapsto \eta(E, \bar{\delta})$ are homogeneous in the bi-limit with degree 1 and $d_{W}+d_{\infty}$ and approximating functions, respectively,

$$
|\bar{\delta}|^{d_{W}}, \frac{c_{1}}{4 n \overline{\mathfrak{\imath}}} W_{0}(E)-\frac{\partial W_{0}}{\partial e_{i}}(E)\left[k_{i, 0}\left(e_{1}+\bar{\delta}\right)-k_{i, 0}\left(e_{1}\right)\right]
$$

and

$$
\begin{aligned}
|\bar{\delta}|^{\frac{d_{W}+d_{\infty}}{d_{W}}}, \frac{c_{1}}{4 n \overline{\mathcal{\imath}}} W_{\infty}(E)^{\frac{d_{W}+d_{\infty}}{d_{W}}} & \\
& -\frac{\partial W_{\infty}}{\partial e_{i}}(E)\left[k_{i, \infty}\left(e_{1}+\bar{\delta}\right)-k_{i, \infty}\left(e_{1}\right)\right] .
\end{aligned}
$$

Hence (see (Andrieu et al., 2008-SICON, Lemma 2.13)) there exists a positive real number $c_{4}$ satisfying

$$
\begin{aligned}
& \frac{\partial W}{\partial e_{i}}(E)\left[k_{i}\left(e_{1}+\bar{\delta}\right)-k_{i}\left(e_{1}\right)\right] \leq \\
& \frac{c_{1}}{4 n \overline{\mathfrak{A}}}\left[W(E)+W(E)^{\frac{d_{W}+d_{\infty}}{d_{W}}}\right]+\frac{c_{4}}{n}\left[|\bar{\delta}|^{d_{W}}+|\bar{\delta}|^{\frac{d_{W}+d_{\infty}}{r_{\infty}, 1}}\right]
\end{aligned}
$$

By letting $\bar{\delta}=-\frac{\delta_{y}}{L^{b}}$, this yields :

$$
\begin{aligned}
T_{y} \leq \frac{c_{1}}{4} \mathfrak{a}(y)\left[W(E)+W(E)^{\frac{d_{W}+d_{\infty}}{d_{W}}}\right] & \\
& +c_{4} \overline{\mathfrak{A}} \mathfrak{a}(y)\left[\left|\frac{\delta_{y}}{L^{b}}\right|^{d_{W}}+\left|\frac{\delta_{y}}{L^{b}}\right|^{\frac{d_{W}+d_{\infty}}{r_{\infty}, 1}}\right] .
\end{aligned}
$$

Bounding $T_{G U}$. The function $E \mapsto \frac{\partial W}{\partial E}(E) \mathfrak{B} E$ is homogeneous in the bi-limit with associated weights $r_{0}$ and $r_{\infty}$ and degrees $d_{W, 0}=d_{W, \infty}=d_{W}$. Hence (see (Andrieu et al., 2008-SICON, Corollary 2.15)) there exists a positive real number $c_{5}$ satisfying : 


$$
\frac{\partial W}{\partial E}(E) \mathfrak{B} E \leq c_{5} W(E) .
$$

With (20) and the definition of $\dot{L}$ in (8), this yields :

$T_{G U} \leq-c_{2} \varphi_{3} \frac{\Omega(u, y, \hat{x})}{L} W(E)+c_{5} \varphi_{1} W(E)$.

Bounding $T_{N L}$. With (6), (23) and (18) give :

$$
\begin{aligned}
\left|\mathfrak{D}_{i}(L)\right| \leq \Omega(u, y, \hat{x}) & \sum_{j=2}^{i} L^{j-1-i}\left|e_{j}\right| \quad(28) \\
& +c_{\infty} L^{-i-b} \sum_{j=2}^{i}\left|L^{b+j-1} e_{j}\right|^{\frac{r_{\infty, i}+d_{\infty}}{r_{\infty}}} .
\end{aligned}
$$

With the inequalities (21) and (18), we know there exists a strictly positive real number $\epsilon_{1}$ satisfying :

$$
\varphi_{2}^{-\epsilon_{1}} \geq L^{-\epsilon_{1}} \geq L^{(b+j-1) \frac{r_{\infty, i}+d_{\infty}}{r_{\infty, j}}-i-b} \quad \forall L \geq \varphi_{2} \geq 1 .
$$

Consequently, for all $L \geq \varphi_{2} \geq 1$, we have :

$$
\left|\mathfrak{D}_{i}(L)\right| \leq \frac{\Omega(u, y, \hat{x})}{L} \sum_{j=2}^{i}\left|e_{j}\right|+c_{\infty} \varphi_{2}^{-\epsilon_{1}} \sum_{j=2}^{i}\left|e_{j}\right|^{\frac{r_{\infty, i}+d_{\infty}}{r_{\infty}, j}} .
$$

On another hand, since (18) and $0=d_{0} \leq d_{\infty}$ imply

$$
\frac{r_{\infty, i}+d_{\infty}}{r_{\infty, i}} \geq 1 \quad i \in\{1 \ldots n\}
$$

the functions

$$
\begin{array}{r}
E \mapsto\left|\frac{\partial W}{\partial e_{i}}(E)\right|\left|e_{j}\right| \quad \text { and } \\
E \mapsto\left|\frac{\partial W}{\partial e_{i}}(E)\right| \sum_{j=2}^{i}\left|e_{j}\right|+\left|e_{j}\right|^{\frac{r_{\infty, i}+d_{\infty}}{r_{\infty, j}}}
\end{array}
$$

are homogeneous in the bi-limit with weights $r_{\infty}$ and $r_{0}$ and degrees $d_{W}$ and $d_{W}+(j-i) d_{\infty}\left(\leq d_{W}\right)$ and $d_{W}$ and $d_{\infty}+d_{W}$ respectively. Hence (see (Andrieu et al., 2008-SICON, Corollary 2.15)) there exists positive real numbers $c_{6}$ and $c_{7}$ satisfying, for all $E$ in $\mathbb{R}^{n}$ and $i$ in $\{1, \ldots, n\}$,

$$
\begin{aligned}
&\left|\frac{\partial W}{\partial e_{i}}(E)\right|\left|e_{j}\right| \leq \frac{c_{6}}{n^{2}} W(E) \quad \forall j \in\{1, \ldots, i\} \\
&\left|\frac{\partial W}{\partial e_{i}}(E)\right|\left(\sum_{j=2}^{i}\left|e_{j}\right|+\left|e_{j}\right|^{\frac{r_{\infty}, i+d_{\infty}}{r_{\infty}, j}}\right) \\
& \leq \frac{c_{7}}{n}\left(W(E)+W(E)^{\frac{d_{\infty}+d_{W}}{d_{W}}}\right) .
\end{aligned}
$$

This gives

$$
\begin{aligned}
\left|T_{N L}\right| \leq c_{6} & \frac{\Omega(u, y, \hat{x})}{L} W(E) \\
& +c_{\infty} c_{7} \varphi_{2}^{-\epsilon_{1}}\left(W(E)+W(E)^{\frac{d_{\infty}+d_{W}}{d_{W}}}\right) .
\end{aligned}
$$

Bound on $\frac{d W(E)}{d \tau}$. Using inequality (26), and the bounds on $T_{\delta}, \frac{d \tau}{T_{y}}, T_{G U}$ and $T_{N L}$, we obtain :

$$
\begin{gathered}
\frac{d W(E)}{d \tau} \leq\left(c_{6}-c_{2} \varphi_{3}\right) \frac{\Omega(u, y, \hat{x})}{L} W(E) \\
+\left(\frac{c_{3}}{\varphi_{2}^{b}}+\frac{c_{\infty} c_{7}}{\varphi_{2}^{\epsilon_{1}}}+c_{5} \varphi_{1}-\frac{3 c_{1}}{4} \mathfrak{a}(y)\right)
\end{gathered}
$$

$$
\begin{array}{r}
\times\left(W(E)+W(E)^{\frac{d_{W}+d_{\infty}}{d_{W}}}\right) \\
+\frac{c_{3}}{n L^{b}} \sum_{i=1}^{n} \mathfrak{H}_{i}\left(\frac{\delta_{i}}{L^{i}}\right)+\frac{c_{4} \overline{\mathfrak{A}}}{L^{b}} \mathfrak{a}(y)\left[\left|\delta_{y}\right|^{d_{W}}+\left|\delta_{y}\right|^{\frac{d_{W}+d_{\infty}}{r_{\infty}, 1}}\right] .
\end{array}
$$

Therefore, with (5) and $L>\varphi_{2}$, by choosing $\varphi_{2}$ and $\varphi_{3}$ sufficiently large and $\varphi_{1}$ sufficiently small, we get :

$$
\begin{aligned}
& \frac{d W(E)}{d t} \leq-\kappa L W(E)+L U, \\
& \text { where } \kappa=\frac{c_{1} \rho}{2} \text { and } \\
& \begin{aligned}
U(t)= & \frac{c_{3}}{n \varphi_{2}^{b}} \sum_{i=1}^{n} \mathfrak{H}_{i}\left(\frac{\delta_{i}(t)}{L(t)^{2}}\right) \\
& +\frac{c_{4} \overline{\mathfrak{\alpha}} \rho}{\varphi_{2}^{b}}\left[\left|\frac{\mathfrak{a}(y(t)) \delta_{y}(t)}{\rho}\right|^{d_{W}}+\left|\frac{\mathfrak{a}(y(t)) \delta_{y}(t)}{\rho}\right|^{\frac{d_{W}+\infty_{\infty}}{r_{\infty}, 1}}\right] .
\end{aligned}
\end{aligned}
$$

Integrating this inequality, it yields on the time of existence of the solutions :

$$
\begin{aligned}
W(E(t)) \leq \exp \left(-\kappa \int_{s}^{t} L(s)\right) W(E(s))+ \\
\quad \int_{s}^{t} \exp \left(-\kappa \int_{r}^{t} L(u) d u\right) L(r) U(r) d r .
\end{aligned}
$$

And since $L>\varphi_{2} \geq 1$, this implies

$W(E(t)) \leq \exp (-\kappa(t-s)) W(E(s))+\frac{1}{\kappa} \sup _{r \in[s, t]} U(r)$.

The function $W$ being proper, it yields an ISS property between the inputs $\frac{\delta_{i}}{L^{2}}$ 's and $\mathfrak{a}(y) \delta_{y}$ and the state $E$. Hence, inequality (11) follows on the time domain of existence of the solutions from the definition (23) of $e_{i}$.

Behavior of $L$. We can rewrite (9) in ${ }^{3}$ :

$\Omega(u, y, \hat{x})$

$$
\leq \Gamma(u, y)\left(1+\sum_{j=2}^{n}\left|x_{j}\right|^{v_{j}}+L^{v_{j}(b+j-1)}\left|e_{j}\right|^{v_{j}}\right) .
$$

Then, with (22) and $L \geq 1$, we get $\epsilon_{2}>0$ satisfying :

$$
\Omega(u, y, \hat{x}) \leq \Gamma(u, y)\left(1+\sum_{j=2}^{n}\left|x_{j}\right|^{v_{j}}+L^{1-\epsilon_{2}}\left|e_{j}\right|^{v_{j}}\right) .
$$

Consequently (8) gives

$$
\begin{aligned}
\dot{L} \leq & L\left(\varphi_{1} \varphi_{2}-\varphi_{1} L+\varphi_{3} \Gamma(u, y)\right. \\
& \left.+\varphi_{3} \Gamma(u, y) \sum_{j=2}^{n}\left|x_{j}\right|^{v_{j}}+L^{1-\epsilon_{2}}\left|e_{j}\right|^{v_{j}}\right) . \\
\leq & -\frac{\varphi_{1}}{2} L^{2} \\
- & L\left(\frac{\varphi_{1}}{4} L-\varphi_{1} \varphi_{2}-\varphi_{3} \Gamma(u, y)-\varphi_{3} \Gamma(u, y) \sum_{j=2}^{n}\left|x_{j}\right|^{v_{j}}\right. \\
& \left.-\left(\frac{4 n \varphi_{3}}{\varphi_{1}}\right)^{\frac{1}{\epsilon_{2}}} \Gamma(u, y)^{\frac{2}{\epsilon_{2}}}-\left(\frac{4 n \varphi_{3}}{\varphi_{1}}\right)^{\frac{1}{\epsilon_{2}}} \sum_{j=2}^{n}\left|e_{j}\right|^{\frac{2 v_{j}}{\epsilon_{2}}}\right) .
\end{aligned}
$$

\footnotetext{
${ }^{3}$ Since $v_{j}$ is smaller than 1 , we have, for all $(a, b) \in \mathbb{R}_{+*}^{2}$, $\frac{a}{\left(a^{v_{j}}+b^{v_{j}}\right)^{\frac{1}{v_{j}}}} \leq \frac{a^{v_{j}}}{a^{v_{j}}+b^{v_{j}}} \leq 1$ and $(a+b)^{v_{j}} \leq a^{v_{j}}+b^{v_{j}}$. 
This implies the existence of a class $\mathcal{K} \mathcal{L}$ function $\beta_{1}$ and a class $\mathcal{K}$ function $\gamma_{1}$ such that, along any solution, we have on its domain of existence

$$
\begin{aligned}
\max \{L(t)- & \left.4 \varphi_{2}, 0\right\} \leq \beta_{1}(L(0), t) \\
& +\sup _{s \leq t} \gamma_{1}(|(\Gamma(u(s), y(s)), x(s), E(s))|) .
\end{aligned}
$$

Hence having a cascade of two ISS systems, (12) and (11) holds on $[0, \mathcal{T})$.

\section{Conclusion}

We have presented a modification of the classical high gain observer with the introduction of a gain updating and of a homogeneous in the bi-limit correction term. We have shown that this extends the domain of applicability and proved convergence for bounded solutions. We have also shown by means of an example, that the modification may improve performance by allowing a better fit of the incremental rate of the nonlinearities.

This work has been performed while the first author was a member of the CAP Group of Imperial College London.

\section{References}

V. Andrieu, L. Praly and A. Astolfi, Proof of Theorem 2, Archive HAL, 00229705, January 2008.

V. Andrieu, L. Praly and A. Astolfi, Homogeneous approximation, recursive observer design and output feedback. SIAM J. Control Optim. Vol. 47, n. 4, pp. 1814-1850, 2008.

A. Astolfi and L. Praly, Global complete observability and output-to-state stability imply the existence of a globally convergent observer. Mathematics of Control, Signals and Systems (2006) 18: 32-65.

J.P. Gauthier, H. Hammouri and S. Othman, A simple observer for nonlinear systems. Application to bioreactors, IEEE Transactions on Automatic Control, Vol. 37, No. 6, June 1992.

J.P. Gauthier and I. Kupka, Deterministic Observation Theory And Applications, Cambridge University Press. 2001

P. Krishnamurthy, F. Khorrami, and R. S. Chandra, Global high-gain based observer and backstepping controller for generalized output-feedback canonical form, IEEE Transactions on Automatic Control, Vol. 48, No. 12, December 2003.

P. Krishnamurthy and F. Khorrami, Dynamic high-gain scaling : state and output feedback with application to systems with ISS appended dynamics driven by all states. IEEE Transactions on Automatic Control, Vol. 49, No. 12, December 2004

H. Lei, J. F. Wei and W. Lin, A global observer for observable autonomous systems with bounded solution trajectories, Proceedings of the 44th IEEE Conference on Decision and Control and the European Control Conference 2005, December 2005.

F. Mazenc, L. Praly, and W. P. Dayawansa, Global stabilization by output feedback : Examples and Counter-Examples, Systems \& Control Letters 23 (1994) 119-125

L. Praly, Asymptotic stabilization via output feedback for lower triangular systems with output dependent incremental rate.
IEEE Transactions on Automatic Control, Vol. 48, No. 6, June 2003.

C. Qian, A Homogeneous Domination Approach for Global Output Feedback Stabilization of a Class of Nonlinear Systems, Proceedings of the 2005 American Control Conference Page(s):4708 - 4715 vol. 7 June 2005. 